\title{
TNFR-1 on tumor cells contributes to the sensitivity of fibrosarcoma to chemotherapy
}

\author{
Jingjing Deng ${ }^{1,2}$, Xiaopu Zhao ${ }^{1,2}$, Lijie Rong ${ }^{1,2}$, Xiao Li $^{1}$, Xiaoman Liu ${ }^{1}$, Zhihai Qin ${ }^{1 凶}$ \\ ${ }^{1}$ Key Laboratory of Protein and Peptide Pharmaceuticals; Chinese Academy of Sciences-University of Tokyo Joint Laboratory of \\ Structural Virology and Immunology, Institute of Biophysics, Chinese Academy of Sciences, Beijing 100101, China \\ ${ }^{2}$ University of Chinese Academy of Sciences, Beijing 100049, China \\ $\triangle$ Correspondence: zhihai@ibp.ac.cn
}

Received January 23, 2013 Accepted March 19, 2013

\begin{abstract}
Impaired tumor necrosis factor receptor-1 (TNFR-1) signaling has been found in some malignant tumors with poor prognosis. However, the exact role of TNFR-1 signaling in fibrosarcoma remains unclear. Here, we explored the question by comparing the growth of TNFR-1 deficient (Tnfr1 ${ }^{-}$) and TNFR-1 competent $\left(\right.$Tnfr $^{+}{ }^{+}$) fibrosarcoma FB61 cells (FB61-m and FB61-R1) in mice. TNFR-1 expression on fibrosarcoma cells delayed their growth in vivo but not in vitro. Moreover, reduced FB61-R1 tumor growth was also obtained in TNFR-1 knockout mice. The mechanism relies mainly on the TNFR-1-mediated downregulation of vascular endothelial growth factor (VEGF) production by tumor cells. Importantly, treatment of FB61-m tumors with melphalan resulted in a short delay of tumor growth, followed by a quick remission. However, when FB61-R1 tumors were treated with melphalan, tumor growth was similarly delayed at first and then completely rejected. Our results reveal evidence for TNFR-1 on tumor cells as a prerequisite in chemotherapy for fibrosarcoma, and provide novel insight into the therapeutic approach against some types of tumors using TNFR-1 angonist.
\end{abstract}

\section{KEYWORDS TNFR-1, fibrosarcoma, chemotherapy}

\section{INTRODUCTION}

Tumor necrosis factor-alpha (TNF- $\alpha$ ), produced by activated macrophages, $T$ cells and several other cell types, is a cytokine that mediates a wide range of biologic activities including the induction of tumor necrosis (Wu et al., 2006; Balkwill, 2009). In many experimental and human cancers, TNF- $\alpha$ is an important component of the interplay between tumor cells and tumor stromal cells (Blankenstein et al., 1991; Qin et al., 1993; Mocellin et al., 2005; Qin et al., 2007; Li et al., 2012). The TNF- $\alpha$ activity has been reported to be closely associated with the disease progress in most patients with cancers as diverse as prostate cancer, breast cancer, renal cell cancer, non-small cell lung cancer, and other tumor types (Balkwill, 2009). Fibrosarcoma is a cancerous tumor derived from fibrous connective tissue and composed of proliferating fibroblasts. This type of malignant cancer most commonly affects the limbs and the trunk of the body, but it can also invade other tissues (Coffin and Alaggio, 2012). The role of TNF- $\alpha$ in fibrosarcoma has not been completely defined.

TNF- $\alpha$ has two cell surface receptors: TNFR-1 and TNFR-2 (Balkwill, 2006). TNFR-1 is a 55-kDa protein which presents a death domain on its intracellular region. It is expressed by almost all cell types and is the major signaling receptor for TNF- $\alpha$ (Cabal-Hierro and Lazo, 2012). Studies showed that a huge majority of myeloma cell lines failed to show TNFR-1 expression (Rauert et al., 2011); impaired TNFR-1 signaling pathway was associated with prostate cancer progression (Wang et al., 2009); infrequent presence of TNFR-1 was observed in ovarian carcinoma effusions in patients (Dong et al., 2008); and a high TNFR-1 expression in Dukes' stage C colorectal cancer could be a favorable indicator of prognosis (Yoshimura et al., 2003). All these indicate that TNFR-1 may play a crucial role in tumor development. It is also reported that an up-regulation of TNFR-1 was found in the tumor biopsies of cancer patients after treatment (van Horssen et al., 2006). Thus, it is important to clarify the possible relations of TNFR-1 expression on fibrosarcoma and their sensitivity to clinical treatment, such as chemotherapy.

The specific outcome of TNFR-1 signaling pathway is tumor type-dependent (Balkwill, 2006, 2009). It is reported that TNF- $\alpha$ activation of TNFR-1 stimulates both pro- and anti-apoptotic 
signaling pathways (Edelblum et al., 2008; Cabal-Hierro and Lazo, 2012). TNFR-1 is able to induce the extrinsic apoptosis signaling pathway upon ligand binding, and transduce signals that promote cell death by apoptosis. Other evidence suggests that TNFR-1 has nonapoptotic functions as well, including promoting cell survival and proliferation, mediating tumorigenesis and tumor progression, in concert with nuclear factor-kB (NF-KB), mitogen-activated protein kinase (MAPK), and Akt signaling (Rivas et al., 2008; Schutze et al., 2008). However, the direct evidence is required to delineate the role of TNFR-1 signaling in fibrosarcoma cells.

In this study we show that TNFR-1 expression on fibrosarcoma is required for the tumor rejection. Interestingly, TNFR-1 does not affect the tumor cell viability, but reduces VEGF production, indicating a novel effect of TNF- $\alpha$ on fibrosarcoma cells. Most importantly, we provide the first evidence for TNFR-1 on tumor cells as a prerequisite in chemotherapy for fibrosarcoma.

\section{RESULTS}

TNFR-1 abundance on fibrosarcoma cells correlates with its tumorigenic potential

The impaired TNFR-1 signaling in tumor cells and the malignant tumor progress have been observed in cancer patients (Yoshimura et al., 2003; Dong et al., 2008). To detect whether TNFR-1 expression on fibrosarcoma cells correlates with their growth, we examined the growth rates and TNFR-1 expression levels of the methylcholanthrene (MCA)-induced primary tumor cells and these cells after passage in immunocompetent mice. The parental FA09 (FA09-0) was weak in terms of tumorigenic potential, only leading to $60 \%$ tumor-positive mice 45 days after tumor inoculation (Fig. 1A). After one passage in BALB/c mice, the tumorigenic potential of these cells (FA09-1) was markedly increased and all mice were tumor bearing 45 days after tumor cell inoculation (Fig. 1A).

To assess whether TNFR-1 confers fibrosarcoma cells with some growth advantage, we examined the TNFR-1 expression levels on FA09-0 and FA09-1 cells using flow cytometry and western blot. As shown in Fig. 1B and 1C, the cell surface expression of TNFR-1 on FA09-1 cells was significantly decreased as compared with that on FA09-0 cells, suggesting TNFR-1 low-expressing fibrosarcoma cells may be selected for tumor growth advantage in vivo.

\section{Establishment of $\operatorname{Tnfr}^{+}$and $T n f r 1^{-}$fibrosarcoma cell lines}

To further investigate the effect of TNFR-1 on fibrosarcoma cells, we established a TNFR-1 deficient tumor cell line FB61 induced by MCA in TNFR-1 knockout BALB/c mice (Zhao et al., 2012). FB61 tumor cells were transfected with a TNFR-1-expressing plasmid as described in materials and methods, resulting in a TNFR-1 competent tumor cell line FB61-R1, with FB61-m as control. TNFR-1 mRNA expression in FB61-R1 or FB61-m cells was shown in Fig. 2A. A 1624-bp band was amplified from FB61-R1 but not FB61-m cells

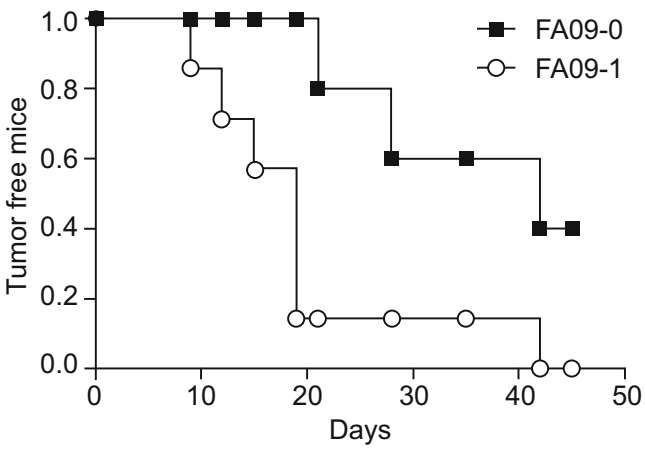

B

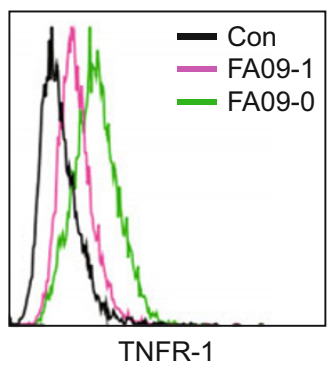

C

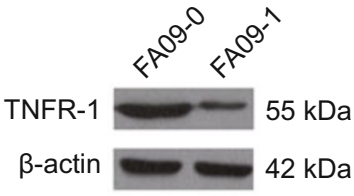

Figure 1. Reduced TNFR-1 expression on FA09 cells correlates with enhanced tumorigenic potential after in vivo passage. (A) Naïve BALB/c mice ( $n=5-7)$ were subcutaneously (s.c.) injected with $2 \times 10^{6}$ FA09-0 (parental FA09) or FA09-1 (one passage in BALB/c mice) cells. Tumor growth was monitored every 3-4 days. The percentages of tumor-free mice were given at the indicated time points. Mice bearing tumors with $>8 \mathrm{~mm}$ in size were recorded as tumor-positive. Experiments were repeated at least three times and representative results are shown. (B) Cell surface expression of TNFR-1 on FA09 cells was reduced after in vivo passage. FA09-0 and FA09-1 cells were stained with antiTNFR-1 mAb or an isotype matched control mAb, then analyzed for TNFR-1 expression by flow cytometry. The histogram overlays show TNFR-1 expression on different cells. (C) Protein levels of TNFR-1 (55 kDa) from FA09-0 and FA09-1 cells were detected by western blot, with $\beta$-actin $(42 \mathrm{kDa})$ as internal control.

(Fig. 2A). Subsequently, we examined TNFR-1 expression on the cell surface by western blot and flow cytometry. Consistent with the previous observation, TNFR-1 was detected only on FB61-R1 cells, but not on FB61-m cells (Fig. 2B and 2C). The extracellular domain of TNFR-1 also exists as a soluble molecule formed via shedding from the cell surface (Levine, 2008). As shown in Fig. 2D, the soluble TNFR-1 expression level in the supernatant of FB61-m can barely be detected, while it reached $58 \mathrm{pg} / \mathrm{mL}$ in the FB61-R1 group. What is more, TNFR-1 on the surface of FB61-R1 cells was functional because exposure of these cells to TNF- $\alpha$ efficiently up-regulated their major histocompatibility complex (MHC) class I expression (Fig. 2E). However, TNF- $\alpha$ did not change the expression of MHC class I molecules on FB61-m cells, as determined by cul- 
A

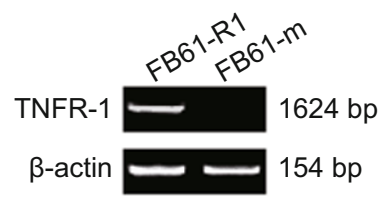

B

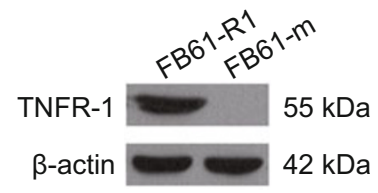

$\mathrm{F}$

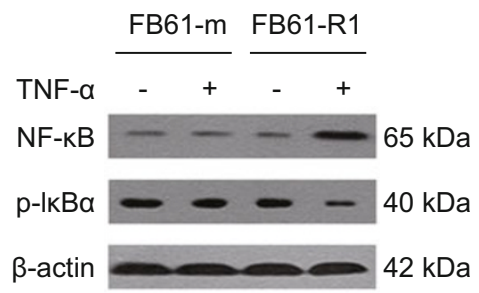

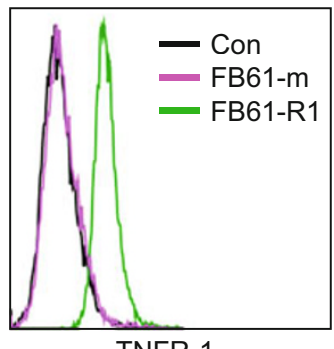

TNFR-1

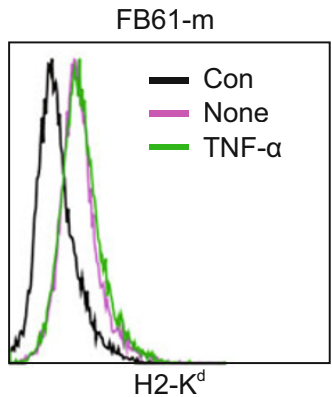

D
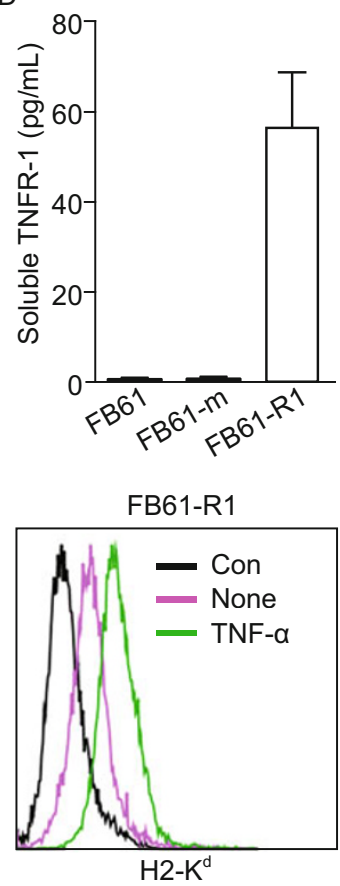

Figure 2. Establishment of TNFR-1 deficient and competent fibrosarcoma cell lines. (A) TNFR-1 gene was transfected into the FB61 cells, resulting in FB61-R1 cells, with FB61-m cells as control. Total RNA was extracted from FB61-m and FB61-R1 cells. TNFR-1 expression was determined by RT-PCR (1624 bp), using the $\beta$-actin gene as control. (B) Protein levels of TNFR-1 from FB61-m and FB61-R1 cells were detected by western blot, with $\beta$-actin as internal control. (C) FB61-m and FB61-R1 tumor cells were stained with the anti-TNFR-1 mAb or an isotype-matched control mAb, and then analyzed by flow cytometry. (D) The supernatants of FB61, FB61-m and FB61-R1 cells were collected, and sTNFR-1 levels were determined by ELISA. The ELISA detected both free and TNF- $\alpha$-binding sTNFR-2 forms. (E) FB61-m and FB61-R1 tumor cells were cultured for $48 \mathrm{~h}$ in the absence (pink) or presence of $20 \mathrm{ng} / \mathrm{mL}$ TNF- $\alpha$ (green) and stained with $\mathrm{PE}$-labeled $\mathrm{mAb}$ against $\mathrm{H}-2 \mathrm{~K}^{\mathrm{d}}$. A PE-labeled isotype-matched $m A b$ was used as control (black). (F) TNF- $\alpha$ stimulation increased the nuclear NF-kb expression, and inactivated IkBa in FB61-R1 cells. FB61-m and FB61-R1 were cultured with or without the stimulation of TNF- $\alpha$ for 20 min. Protein levels of nuclear NF-kb $(65 \mathrm{kDa})$ and $\mathrm{p}-\mathrm{I} \mathrm{kBa}(40 \mathrm{kDa})$ were detected by western blot, with $\beta$-actin as internal control.

turing these cells in the presence of recombinant mouse TNF- $\alpha$ for $48 \mathrm{~h}$. The signaling pathway downstream of TNFR-1 was also detected. TNF- $\alpha$ binding TNFR-1 could induce degradation of inhibitor kappa B-alpha ( $(\mathrm{KB} \alpha)$ in cells, resulting in the release of NF-KB, which then translocates to the nucleus (CabalHierro and Lazo, 2012). As shown in Fig. 2E, IKBa was only reduced in TNF- $\alpha$-treated FB61-R1 cells (Fig. 2F). Similarly, the protein level of nuclear NF-KB was only increased in TNFa-treated FB61-R1 cells (Fig. 2F). These results confirm that TNFR-1 is successfully reconstructed in fibrosarcoma cell line FB61.

TNFR-1 expression on fibrosarcoma cells leads to the delayed tumor growth

In order to evaluate the effect of TNFR-1 on fibrosarcoma cells in tumor growth, FB61-m and FB61-R1 cells were correspondingly inoculated into the left and right flanks of BALB/c mice. The tumor growth rate results showed that TNFR-1 expression on fibrosarcoma cells significantly reduced the FB61-R1 tumor size no matter at the dose of $1 \times 10^{5}$ cells (Fig. $3 \mathrm{~A}$ ) or $5 \times 10^{5}$ cells (Fig. 3B). One of the FB61-R1 tumors was even rejected at 20 days after tumor inoculation at the dose of $5 \times 10^{5}$ cells. Apart from FB61 fibrosarcoma, the similar results were also obtained from FD99 fibrosarcoma (data not shown).

To explore whether the FB61-R1 tumor growth retardation is due to the reduced survival rate of these cells, FB61-m and FB61-R1 cells were analyzed for cell survival rate at $72 \mathrm{~h}$ with or without the stimulation of mouse TNF- $\alpha$ in vitro. As shown in Fig. 3C, TNF- $\alpha$ had little influence on the survival rate of FB61$\mathrm{m}$ or FB61-R1 cells. Taken together, these results show that TNFR-1 expression on fibrosarcoma cells leads to the delayed tumor growth in vivo, but does not affect the cell viability in vitro.

Delayed tumor growth of $\operatorname{Tnfr}^{+}$fibrosarcoma cells is also observed in TNFR-1 deficient mice

TNFR-1 is expressed on almost all cell types in wild type mice (Aggarwal, 2003). Thus besides fibrosarcoma cells, many 


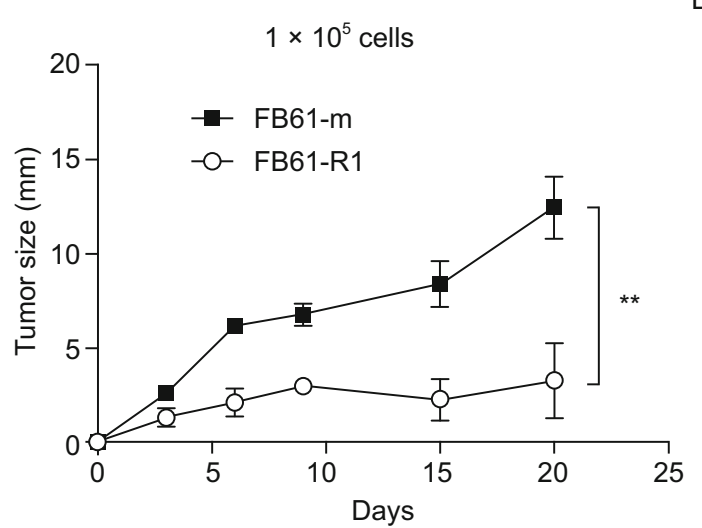

B

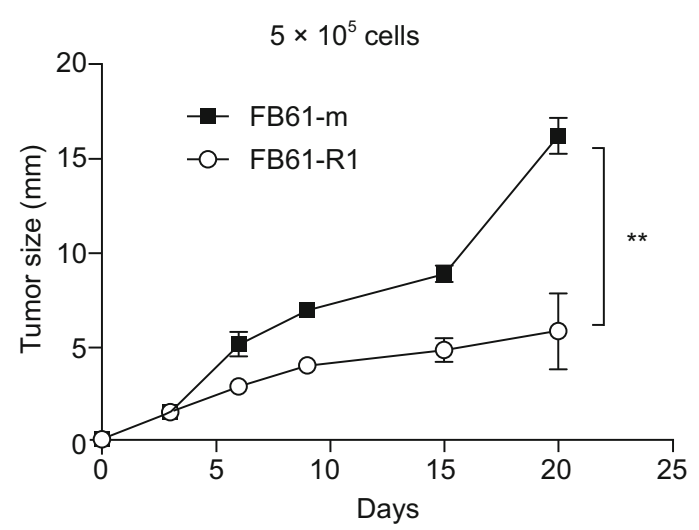

C

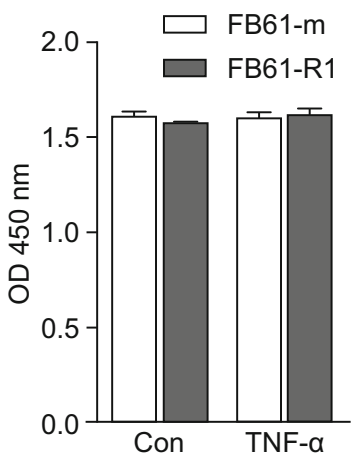

Figure 3. TNFR-1 expression on fibrosarcoma cells retards tumor growth in vivo. FB61-m ( $\bullet$ ) and FB61-R1 (o) cells were s.c. inoculated to the left and right flanks of BALB/c mice $(n=4-7)$ at the amount indicated (A, $\left.1 \times 10^{5} ; \mathrm{B}, 5 \times 10^{5}\right)$. Tumor growth was monitored every $3-5$ days. Experiments were repeated at least three times and representative results are shown. (C) FB61-m and FB61-R1 cells were inoculated into 96-well plates in the presence of $0,20 \mathrm{ng} / \mathrm{mL}$ mouse TNF- $\alpha$. Cell survival rate was determined using a CCK-8 kit at $72 \mathrm{~h}$ after inoculation. Absorbance values detected at $450 \mathrm{~nm}$. other types of cells, especially the tumor stromal cells can respond to TNF- $\alpha$ in this model. The TNFR-1 deficient mice were then used to exclude the effects of TNFR-1 on stromal cells. FB61-m and FB61-R1 cells were inoculated to the TNFR-1 deficient mice as described before. We found that the tumor size of FB61-R1 was also significantly shrunk compared with the FB61-m group in TNFR-1 knockout mice (Fig. 4). Taken together, these results show that the response of $\mathrm{Tnfr}^{+}$fibrosarcoma cells to endogenous TNF- $\alpha$ impairs the tumor growth.

\section{TNFR-1 activation inhibits VEGF production by} fibrosarcoma cells

Next, we investigated whether TNFR-1 activation on fibrosarcoma cells impairs tumor angiogenesis. The concentration of hemoglobin in the tumor was quantified to reflect the abundance of blood vessels. As shown in Fig. 5A, the FB61-m tumors had $7.14 \pm 1.77 \mu \mathrm{g} / \mathrm{mg}$ hemoglobin, whereas the hemoglobin concentration of FB61-R1 tumors was only $2.13 \pm 0.25$ $\mu \mathrm{g} / \mathrm{mg}$. Immunostaining using an anti-mouse CD31 monoclonal antibody (mAb) was also performed on sections of these tumors to detect the vasculature intensity. Consistent with the results above, a decreased intensity of $\mathrm{CD} 31^{+}$microvessels was observed in FB61-R1 tumors compared with the FB61$\mathrm{m}$ tumors (Fig. 5B and $5 \mathrm{C}$ ). The findings suggest that TNFR-1 expression on fibrosarcoma cells inhibits tumor-induced angiogenesis.

We examined subsequently how TNFR-1 expression on fibrosarcoma cells mediates its effect for angiostasis. VEGF has been shown to play a central role in tumor neovascularization, which is mainly produced by tumor cells (Ferrara et al., 2003). To investigate whether TNFR-1 activation alters the production of VEGF by fibrosarcoma cells, FB61-R1 and FB61$m$ cells were stimulated with TNF- $\alpha$ in vitro and the mRNA of

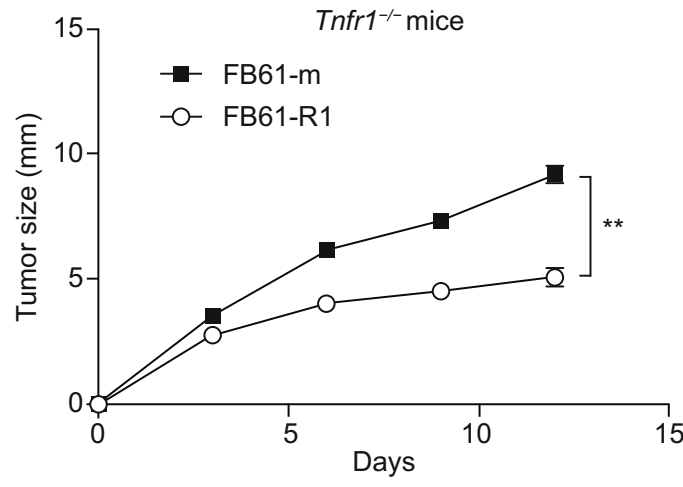

Figure 4. Delayed tumor growth of $\operatorname{Tnfr}^{+}$fibrosarcoma cells is also observed in TNFR-1 deficient mice. TNFR-1 knockout mice $(n=8)$ were s.c. inoculated with $5 \times 10^{5} \mathrm{FB} 61-\mathrm{m}(\mathbf{m})$ or FB61-R1 (o) cells at the left or right flanks respectively. The tumor growth of different groups was monitored as described in Fig. 3A. Experiments were repeated at least three times and representative results are shown. 
A

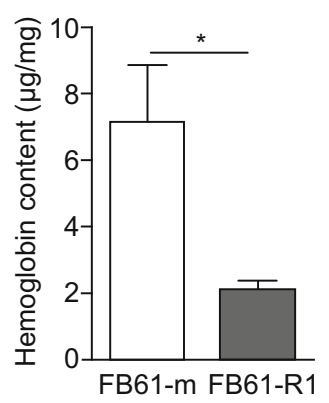

B

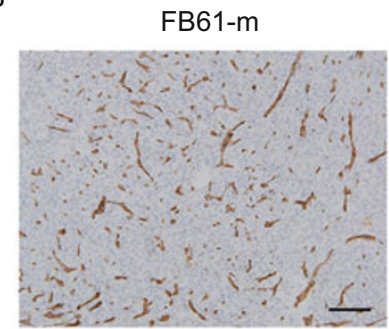

C

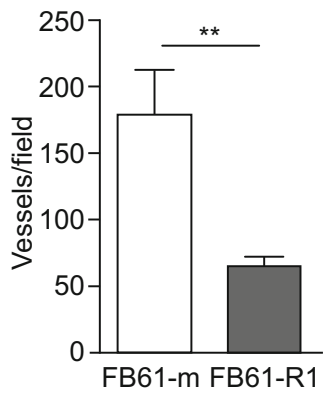

FB61-R1

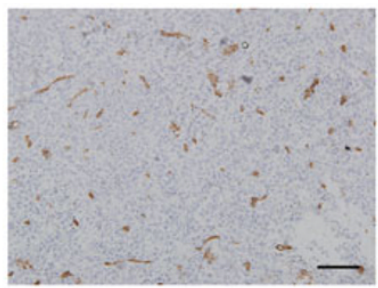

Figure 5. TNF- $\alpha$ acting on $\mathrm{Tnfr}^{+}$fibrosarcoma cells inhibits tumor angiogenesis. (A) FB61-m (white bar) and FB61-R1 (black bar) cells were inoculated to the BALB/c mice as described in Fig. 3B. Twenty days later, the hemoglobin content in tumors was measured using the Drabkin's procedure. (B) Tumor sections in panel A were stained with a rat anti-mouse CD31 mAb to demarcate the vascular. Scale bar represents $100 \mu \mathrm{m}$. (C) Statistics regarding the microvessel density of the different tumor sections described in B was measured according to Weidner's methods.

both cells was quantified. The mRNA of VEGF in FB61-R1 cells was clearly down-regulated following TNF- $\alpha$ treatment, while TNF- $\alpha$ was unable to affect the mRNA expression level of VEGF in FB61-m cells (Fig. 6A). VEGF concentration from the supernatant of fibrosarcoma cells was also determined using ELISA. As shown in Fig. 6B, VEGF production was significantly inhibited when $\mathrm{Tnfr}^{+}{ }^{+}$fibrosarcoma cells respond to TNF- $\alpha$. In summary, the results indicate that TNF- $\alpha$ reacting on $\mathrm{Tnfr}^{+}$fibrosarcoma cells inhibits tumor angiogenesis possibly via down-regulation of tumor cell-derived VEGF.

\section{$\mathrm{Tnfr}^{+}{ }^{+}$fibrosarcoma is more sensitive to chemotherapy}

Chemotherapy is an effective way to treat many types of cancer, and melphalan has been used for cancer treatments since 1966 (Palumbo et al., 2006; Sha et al., 2012). The correlation of TNFR-1 expression on tumor cells and the chemotherapy efficiency for fibrosarcoma was further investigated. As shown in Fig. 7A, melphalan treatment resulted in a short delay of tumor growth, but a quick remission of FB61-m tumors. Importantly, when FB61-R1 tumors were treated with melphalan, tumor growth was similarly delayed at first and then completely rejected at 32 days after tumor inoculation (Fig. 7B).

In summary, these data clearly demonstrate that TNFR-1

expression on fibrosarcoma cells inhibits tumor growth, and it largely contributes to the sensitivity of chemotherapy.

\section{DISCUSSION}

In this study, the direct effects of TNF- $\alpha$ on fibrosarcoma cells were modeled using cancer cells with or without TNFR-1 expression. TNFR-1 expressing FB61 cells were insensitive to the cytotoxicity of TNF- $\alpha$ in vitro. But VEGF production was reduced in fibrosarcoma cells after TNFR-1 activation. Further in vivo experiments showed that TNFR-1 expression on fibrosarcoma cells retarded tumor growth, and contributed to the sensitivity of chemotherapy. Therefore, our finding provides evidence that TNFR-1 expression on fibrosarcoma facilitates tumor rejection in chemotherapy. It suggests that a high TNFR-1 expression on tumor cells could be a favorable indicator of prognosis and TNFR-1 may be an effective target in chemotherapy for some tumor types in the clinic.

Fibrosarcoma, originating from mesenchymal cell, is composed of malignant fibroblasts in a collagen background (Coffin and Alaggio, 2012). It is a malignant cancer with about $15 \%$ mortality rate (Orbach et al., 2005; Orbach et al., 2010; Coffin and Alaggio, 2012). TNFR-1 signaling in tumor growth has been largely studied. In other studies, it mainly focused on the direct effect of TNFR-1 on tumor cell survival or apoptosis

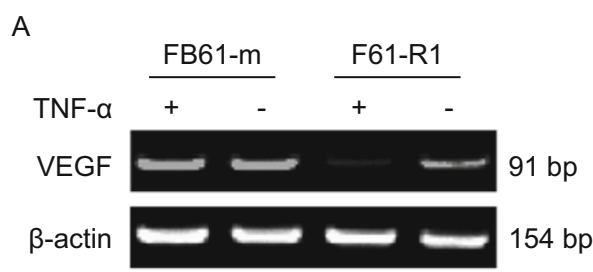

B

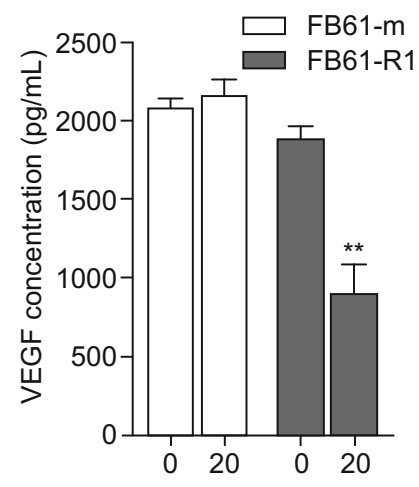

Figure 6. TNFR-1 activation inhibits VEGF production by fibrosarcoma cells. (A) FB61-m and FB61-R1 cells were cultured for $6 \mathrm{~h}$ in serum-free medium with or without the stimulation of TNF- $\alpha(20 \mathrm{ng} / \mathrm{mL})$. The mRNA expression of VEGF (91 bp) was determined by RT-PCR, normalized to $\beta$-actin content. (B) FB61$\mathrm{m}$ and FB61-R1 cells were stimulated with indicated concentrations of TNF- $\alpha(0,20 \mathrm{ng} / \mathrm{mL})$. VEGF concentration in the supernatants of tumor cells was detected by ELISA at $24 \mathrm{~h}$. 


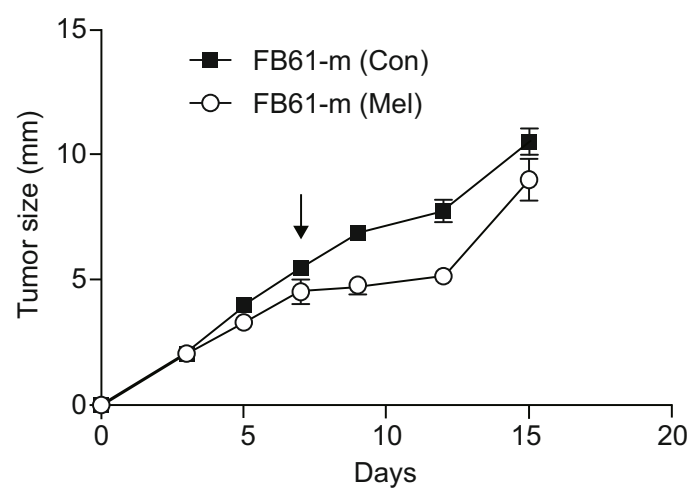

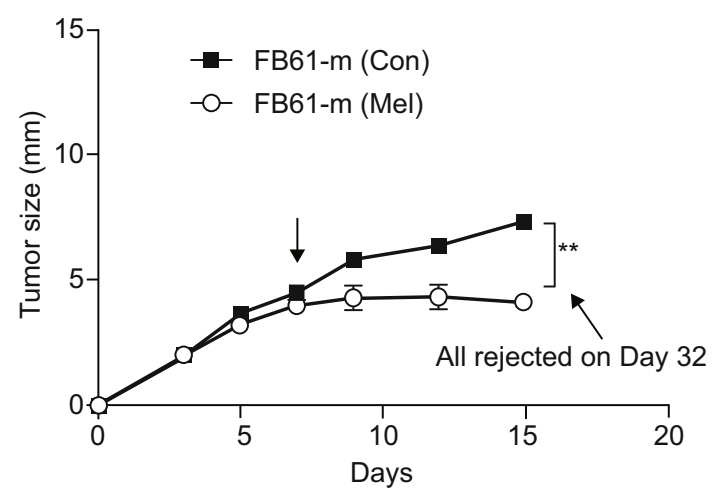

Figure 7. Tnfr1 $^{+}$fibrosarcoma cells are more sensitive to chemotherapy. BALB/c mice $(n=6-8)$ were s.c. inoculated with $5 \times 10^{5}$ FB61-m (A) and FB61-R1 (B) cells. At 7 days after tumor inoculation, mice were i.p. injected with melphalan ( 0 ) or not ( $\bullet$ ) as indicated by arrows. The tumor growth of different groups was monitored as described in Fig. 3A. Experiments were repeated at least three times and representative results are shown.

(Edelblum et al., 2008; Rauert et al., 2011). In this study, we firstly demonstrated that TNF- $\alpha$ had little direct effect on fibrosarcoma cell growth, but other multiple effects on fibrosarcoma cells. Our data showed that TNF- $\alpha$ acting on Tnfr1 ${ }^{+}$fibrosarcoma cells down-regulated VEGF production, and induced the angiostasis. In addition, it also up-regulated MHC class I expression on FB61-R cells, which may play a significant role for better $\mathrm{T}$ cell recognition/elimination in this tumor model (Qin and Blankenstein, 2000). In particular, the enhanced NF-KB activity in FB61-R1 cells may contribute to the tumor cell immunogenicity, for NF-KB families have emerged as critical regulators for cell immunogenic activities (Ouaaz et al., 2002). Furthermore, TNF-related apoptosis-inducing ligand (TRAIL) enables immune effectors to detect and lyse tumor cells (Green et al., 2009; Kepp et al., 2009a; Kepp et al., 2009b), demonstrating a capacity of TNFR-1 in stimulating immunologic tumor cell death. Considering the complex cell-cell interactions in the tumor microenvironment in vivo, it is possible that except for endogenous TNF- $\alpha$, other cytokines may also be involved in the tumor growth deficiency of FB61-R1 (Ruegg et al., 1998; Bauer et al., 2009). Therefore, the exact mechanisms of how $\mathrm{Tnfr}^{+}$fibrosarcoma cells were more efficiently rejected are still required further investigation.

Although surgery should still remain the mainstay of fibrosarcoma treatment, chemotherapy has been reported to be fairly effective especially in infantile fibrosarcoma (IFS) (Orbach et al., 2010). Melphalan is an alkylating agent with broadspectrum activity in a variety of malignancies that creates interand intra-strand DNA cross-links. It has been used for treating cancer patients for decades (Palumbo et al., 2006). However, a variety of side effects come along with chemotherapy treatments including system inflammation induction. Several studies have shown that systemic chemotherapy treatment to cancer patients often stimulates the production of interleukin-1 $\beta$ (IL-1 3 ), TNF- $\alpha$, and IL-6 (Wood et al., 2006a, 2006b). Researchers have also found that various chemotherapeutic agents up-regulate angiogenic VEGF secretion in tumor cells, which had significantly deleterious effect on disease-free survival of patients (Riedel et al., 2004; Ogata et al., 2006; Fan et al., 2008). Thus combination therapy with anti-inflammatory agent is usually used in clinical trials. Melphalan plus dexamethasone (acts as an anti-inflammatory agent) is a common conventional chemotherapy for various disorders (Li et al., 2011). Liver cancer was also effectively treated using a combination of melphalan and bevacizumab (an anti-VEGF antibody) (Voron et al., 2012). In this study, VEGF production was largely reduced in fibrosarcoma cells with TNFR-1 activation. The reduced VEGF may not just contribute to the angiostasis, but also at least partly, counteract with the side effects of chemotherapy.

In conclusion, this study reveals evidence that TNFR-1 expression on fibrosarcoma cells contributes to the tumor rejection especially during chemotherapy, indicating a novel insight into the therapeutic approach against tumors using TNFR1-angonist.

\section{MATERIALS AND METHODS}

\section{Mice}

TNFR-1 knockout and competent mice on BALB/c background were obtained as described previously (Zhao et al., 2012). All mice were bred in the specific pathogen-free barrier facility of Institute of Biophysics, Chinese Academy of Sciences. In all experiments described here, female mice aged 6-8 weeks were used.

\section{Cell lines}

The Tnfr1 ${ }^{-}$fibrosarcoma FB61 cells and the Tnfr ${ }^{-}$cell line FD99 were chose for the experiments (Zhao et al., 2012). Plasmid pLPC-TNFR-1 was transfected into the cells, and the transfectants were screened for TNFR-1 positive cells. As a control, cells were also transfected with the empty vector pLPC, resulting in the mock-transfected cell line. 


\section{Tumor growth assay}

FA09 fibrosarcoma cells are of BALB/c origin and were established in our laboratory the same way as FA10 cells (Li et al., 2008, 2009). FA09-0 is the parental tumor cell, and FA09-1 is the in vivo passaged cell isolated from BALB/c mice which carried tumors arising from FB09-0. To investigate the effects of in vivo passage on tumorigenic potential of tumor cells, BALB/c mice were injected at the right flanks with FA09-0 and at the left flank with FA09-1. Tumors were measured by caliper every $3-4$ days. Mice bearing a tumor of $>8$ $\mathrm{mm}$ in diameter were recorded as tumor positive. To compare the growth rate of nnfr $^{-}$and Tnfr $^{+}$tumors in vivo, FB61-m and FB61$\mathrm{R} 1$ cells were correspondingly injected into the left and right flanks of $\mathrm{BALB} / \mathrm{c}$ mice at the amount indicated. To investigate the role of TNFR-1 in tumor cell sensitivity to chemotherapy, mice were given melphalan $(7.5 \mathrm{mg} / \mathrm{kg}$ ) by intraperitoneal (i.p.) injection when tumor size reached 6-8 $\mathrm{mm}$.

\section{Flow cytometry}

Tumor cells were harvested to form single cell suspension. Cell surface TNFR-1 expression was determined using an anti-mouse TNFR-1 antibody (Ab) (BioLegend). To confirm TNFR-1 function, FB61-m and FB61-R1 cells were cultured with or without $20 \mathrm{ng} / \mathrm{mL}$ recombinant murine TNF- $\alpha$ (Peprotech) for $48 \mathrm{~h}$. These cells were then stained for $\mathrm{MHC}$ class I expression with an anti-H2- $\mathrm{K}^{\mathrm{d}} \mathrm{mAb}$ (BD Pharmingen) or an isotype-matched control mAb.

\section{Cell survival rate}

FB61-R1 and FB61-m cells were seeded into 96-well plates at $3 \times 10^{3}$ per well in $200 \mu \mathrm{L}$ RPMI 1640 with or without the addition of $20 \mathrm{ng} / \mathrm{mL}$ TNF- $\alpha$. After $72 \mathrm{~h}$ of culturing, cell viability was determined using a CCK-8 kit (Beyotime). Absorbance values were measured at $450 \mathrm{~nm}$ on a microplate spectrophotometer (Bio-Rad Laboratories).

\section{Soluble TNFR-1 (sTNFR-1) and VEGF concentration detection}

To detect sTNFR-1 production, the supernatant of FB61-m or FB61-R1 cells was collected after a 48-h culture. The concentration of STNFR-1 in the cell supernatant was determined using an ELISA kit (R\&D Systems, Minneapolis, MN, USA), which detected both free and TNFbinding forms of sTNFR-1.

VEGF production in the tumor tissues was determined using an ELISA kit according to the manufacturer's instructions (eBioscience).

\section{Analysis of hemoglobin content in tumors}

Hemoglobin content in isolated tumors was estimated as described previously (Lu et al., 2009). In brief, tumors were isolated, minced, and homogenized. The hemoglobin in the tumor tissues was then extracted. One milliliter $(\mathrm{mL})$ of the sample was added to $5 \mathrm{~mL}$ of Drabkin's solution. Readings were obtained at $540 \mathrm{~nm}$ using a spectrophotometer. The concentration of hemoglobin in different tumors was expressed as hemoglobin content per microgram of tumor tissue.

\section{Real time (RT)-PCR}

RNA was extracted using the TRIZOL reagent kit per the manufacturer's instructions (Molecular Research Center). Gene expression was detected with the following designed primer pairs: TNFR-1 (sense) 5'-GGAATTCAAGCTTGGCCGGACATGGGTCT-3' and (antisense) 5'-GGAATTCCCGCGGCCTGTCTTTGGCACCCT-3'; VEGF (sense) 5'-CTCCACCATGCCAAG-3' and (antisense) 5'-GCTGGTAGACATCCAT-3'; and $\beta$-actin (sense) 5'-GAAGTGTGACGTTGACATCCGTA-3' and (antisense) 5'-CTCAGGAGGAGCAATGATCTTGA -3'.

\section{Immunostaining and immunoblotting}

For histological staining of tumor tissues, tumors were embedded in optimal cutting temperature (OCT) compound, frozen, and cut with a cryostat. Tumor tissues were stained with a rat anti-mouse CD31 $\mathrm{mAb}$ (BD biosciences), with an isotype-matched control mAb (BD biosciences) as control. Pictures were taken using an Olympus BX51 microscope. Microvessel density was measured according to Weidner's method (Lu et al., 2009).

Nuclear and cytoplasmic protein was extracted using a nuclear and cytoplasmic protein extraction kit (Beyotime). Primary antibodies used were as follows: TNFR-1 (1:1000; Abcam), NF-kB (1:1000; Cell Signaling Technology), p-IkBa (1:1000; Cell Signaling Technology), and $\beta$-actin (1:8000; Sigma-Aldrich). HRP-conjugated goat anti-mouse or goat anti-rabbit lgG (Thermo) were used as secondary antibodies.

\section{Statistical analysis}

Statistical analysis was performed using a two-tailed Student's $t$ test. Statistically significant differences are indicated as follows: *, $P<0.05$; **, $P<0.01$.

\section{ACKNOWLEDGEMENTS}

We would like to thank Junying Jia and Zhenwei Yang for help with flow cytometry and RT-PCR. This work was supported by the Ministry of Science and Technology of China (Grant No. 2012CB917103) and National Natural Science Foundation of China (Grant Nos.81030049 and 91229203).

\section{COMPLIANCE WITH ETHICS GUIDELINES}

Jingjing Deng, Xiaopu Zhao, Lijie Rong, Xiao Li, Xiaoman Liu and Zhihai Qin declare that they have no conflict of interest.

All institutional and national guidelines for the care and use of laboratory animals were followed.

\section{ABBREVIATIONS}

FB61-R1, FB61 with TNFR-1 expression; MCA, methylcholanthrene; NF-kB, nuclear factor-kB; sTNFR-1, soluble TNFR-1; TNFR-1, TNF receptor-1; Tnfr1-' TNFR-1 deficient; Tnfr1 ${ }^{+}$, TNFR-1 competent; VEGF, vascular endothelial growth factor

\section{REFERENCES}

Aggarwal, B.B. (2003). Signalling pathways of the TNF superfamily: a double-edged sword. Nat Rev Immunol 3, 745-756.

Balkwill, F. (2006). TNF-alpha in promotion and progression of cancer. Cancer Metastasis Rev 25, 409-416.

Balkwill, F. (2009). Tumour necrosis factor and cancer. Nat Rev Cancer 9, 361-371.

Bauer, S., Oosterwijk-Wakka, J.C., Adrian, N., Oosterwijk, E., Fischer, 
E., Wuest, T., Stenner, F., Perani, A., Cohen, L., Knuth, A., et al. (2009). Targeted therapy of renal cell carcinoma: synergistic activity of cG250-TNF and IFNg. Int J Cancer 125, 115-123.

Blankenstein, T., Qin, Z.H., Uberla, K., Muller, W., Rosen, H., Volk, H.D., and Diamantstein, T. (1991). Tumor suppression after tumor celltargeted tumor necrosis factor alpha gene transfer. J Exp Med 173, 1047-1052.

Cabal-Hierro, L., and Lazo, P.S. (2012). Signal transduction by tumor necrosis factor receptors. Cell Signal 24, 1297-1305.

Coffin, C.M., and Alaggio, R. (2012). Fibroblastic and myofibroblastic tumors in children and adolescents. Pediatr Dev Pathol 15, 127-180.

Dong, H.P., Kleinberg, L., Silins, I., Florenes, V.A., Trope, C.G., Risberg, B., Nesland, J.M., and Davidson, B. (2008). Death receptor expression is associated with poor response to chemotherapy and shorter survival in metastatic ovarian carcinoma. Cancer 112 , 84-93.

Edelblum, K.L., Goettel, J.A., Koyama, T., McElroy, S.J., Yan, F., and Polk, D.B. (2008). TNFR1 promotes tumor necrosis factor-mediated mouse colon epithelial cell survival through RAF activation of NFkappaB. J Biol Chem 283, 29485-29494.

Fan, F., Gray, M.J., Dallas, N.A., Yang, A.D., Van Buren, G., 2nd, Camp, E.R., and Ellis, L.M. (2008). Effect of chemotherapeutic stress on induction of vascular endothelial growth factor family members and receptors in human colorectal cancer cells. Mol Cancer Ther 7, 3064-3070.

Ferrara, N., Gerber, H.P., and LeCouter, J. (2003). The biology of VEGF and its receptors. Nat Med 9, 669-676.

Green, D.R., Ferguson, T., Zitvogel, L., and Kroemer, G. (2009). Immunogenic and tolerogenic cell death. Nat Rev Immunol 9, 353-363.

Kepp, O., Tesniere, A., Schlemmer, F., Michaud, M., Senovilla, L., Zitvogel, L., and Kroemer, G. (2009a). Immunogenic cell death modalities and their impact on cancer treatment. Apoptosis 14, 364-375.

Kepp, O., Tesniere, A., Zitvogel, L., and Kroemer, G. (2009b). The immunogenicity of tumor cell death. Curr Opin Oncol 21, 71-76.

Levine, S.J. (2008). Molecular mechanisms of soluble cytokine receptor generation. J Biol Chem 283, 14177-14181.

Li, J., Chen, L., and Qin, Z. (2012). Multifaceted tumor stromal fibroblasts. Cancer Microenviron 5, 187-193.

Li, J., Zhang, W., Jiao, L., Duan, M.H., Guan, H.Z., Zhu, W.G., Tian, Z., and Zhou, D.B. (2011). Combination of melphalan and dexamethasone for patients with newly diagnosed POEMS syndrome. Blood $117,6445-6449$.

Li, Z., Chen, L., and Qin, Z. (2009). Paradoxical roles of IL-4 in tumor immunity. Cell Mol Immunol 6, 415-422.

Li, Z., Jiang, J., Wang, Z., Zhang, J., Xiao, M., Wang, C., Lu, Y., and Qin, Z. (2008). Endogenous interleukin-4 promotes tumor development by increasing tumor cell resistance to apoptosis. Cancer Res 68, 8687-8694

Lu, Y., Yang, W., Qin, C., Zhang, L., Deng, J., Liu, S., and Qin, Z. (2009). Responsiveness of stromal fibroblasts to IFN-gamma blocks tumor growth via angiostasis. J Immunol 183, 6413-6421.

Mocellin, S., Rossi, C.R., Pilati, P., and Nitti, D. (2005). Tumor necrosis factor, cancer and anticancer therapy. Cytokine Growth Factor Rev 16, 35-53.

Ogata, Y., Matono, K., Mizobe, T., Ishibashi, N., Mori, S., Akagi, Y., Ikeda, S., Ozasa, H., Murakami, H., and Shirouzu, K. (2006). The expression of vascular endothelial growth factor determines the efficacy of post-operative adjuvant chemotherapy using oral fluoropyrimidines in stage II or III colorectal cancer. Oncol Rep 15, 1111-1116.

Orbach, D., Rey, A., Cecchetto, G., Oberlin, O., Casanova, M., Thebaud, E., Scopinaro, M., Bisogno, G., Carli, M., and Ferrari, A. (2010). Infantile fibrosarcoma: management based on the European experience. J Clin Oncol 28, 318-323.

Orbach, D., Rey, A., Oberlin, O., Sanchez de Toledo, J., Terrier-Lacombe, M.J., van Unnik, A., Quintana, E., and Stevens, M.C. (2005). Soft tissue sarcoma or malignant mesenchymal tumors in the first year of life: experience of the International Society of Pediatric Oncology (SIOP) Malignant Mesenchymal Tumor Committee. J Clin Oncol 23, 4363-4371.

Ouaaz, F., Arron, J., Zheng, Y., Choi, Y., and Beg, A.A. (2002). Dendritic cell development and survival require distinct NF-kappaB subunits. Immunity 16, 257-270.

Palumbo, A., Bringhen, S., Caravita, T., Merla, E., Capparella, V., Callea, V., Cangialosi, C., Grasso, M., Rossini, F., Galli, M., et al. (2006). Oral melphalan and prednisone chemotherapy plus thalidomide compared with melphalan and prednisone alone in elderly patients with multiple myeloma: randomised controlled trial. Lancet 367 , 825-831.

Qin, Y., Auh, S., Blokh, L., Long, C., Gagnon, I., and Hamann, K.J. (2007). TNF-alpha induces transient resistance to Fas-induced apoptosis in eosinophilic acute myeloid leukemia cells. Cell Mol Immunol 4, 43-52.

Qin, Z., and Blankenstein, T. (2000). CD4+ T cell--mediated tumor rejection involves inhibition of angiogenesis that is dependent on IFN gamma receptor expression by nonhematopoietic cells. Immunity 12, 677-686.

Qin, Z., Kruger-Krasagakes, S., Kunzendorf, U., Hock, H., Diamantstein, T., and Blankenstein, T. (1993). Expression of tumor necrosis factor by different tumor cell lines results either in tumor suppression or augmented metastasis. J Exp Med 178, 355-360.

Rauert, H., Stuhmer, T., Bargou, R., Wajant, H., and Siegmund, D. (2011). TNFR1 and TNFR2 regulate the extrinsic apoptotic pathway in myeloma cells by multiple mechanisms. Cell Death Dis 2, e194.

Riedel, F., Gotte, K., Goessler, U., Sadick, H., and Hormann, K. (2004). Targeting chemotherapy-induced VEGF up-regulation by VEGF antisense oligonucleotides in HNSCC cell lines. Anticancer Res 24, 2179-2183.

Rivas, M.A., Carnevale, R.P., Proietti, C.J., Rosemblit, C., Beguelin, W., Salatino, M., Charreau, E.H., Frahm, I., Sapia, S., Brouckaert, P., et al. (2008). TNF alpha acting on TNFR1 promotes breast cancer growth via p42/P44 MAPK, JNK, Akt and NF-kappa B-dependent pathways. Exp Cell Res 314, 509-529.

Ruegg, C., Yilmaz, A., Bieler, G., Bamat, J., Chaubert, P., and Lejeune, F.J. (1998). Evidence for the involvement of endothelial cell integrin alphaVbeta3 in the disruption of the tumor vasculature induced by TNF and IFN-gamma. Nat Med 4, 408-414.

Schutze, S., Tchikov, V., and Schneider-Brachert, W. (2008). Regulation of TNFR1 and CD95 signalling by receptor compartmentalization. Nat Rev Mol Cell Biol 9, 655-662.

Sha, S., Jin, H., Li, X., Yang, J., Ai, R., and Lu, J. (2012). Comparison of caspase-3 activation in tumor cells upon treatment of chemotherapeutic drugs using capillary electrophoresis. Protein Cell 3, 
392-399.

van Horssen, R., Rens, J.A., Brunstein, F., Guns, V., van Gils, M., Hagen, T.L., and Eggermont, A.M. (2006). Intratumoural expression of TNF-R1 and EMAP-II in relation to response of patients treated with TNF-based isolated limb perfusion. Int J Cancer 119, $1481-1490$.

Voron, T., Zinzindohoue, F., Journois, D., Herve, C., Ponzio, O., and Lucas, N. (2012). Hyperthermic isolated liver perfusion with melphalan and bevacizumab. J Visc Surg 150, 60-66.

Wang, D., Montgomery, R.B., Schmidt, L.J., Mostaghel, E.A., Huang, H., Nelson, P.S., and Tindall, D.J. (2009). Reduced tumor necrosis factor receptor-associated death domain expression is associated with prostate cancer progression. Cancer Res 69, 9448-9456.

Wood, L.J., Nail, L.M., Gilster, A., Winters, K.A., and Elsea, C.R. (2006a). Cancer chemotherapy-related symptoms: evidence to suggest a role for proinflammatory cytokines. Oncol Nurs Forum
33, 535-542.

Wood, L.J., Nail, L.M., Perrin, N.A., Elsea, C.R., Fischer, A., and Druker, B.J. (2006b). The cancer chemotherapy drug etoposide (VP-16) induces proinflammatory cytokine production and sickness behavior-like symptoms in a mouse model of cancer chemotherapy-related symptoms. Biol Res Nurs 8, 157-169.

Wu, X., Wei, H., Zhang, J., and Tian, Z. (2006). Increased uterine NKderived IFN-gamma and TNF-alpha in C57BL/6J mice during early gestation. Cell Mol Immunol 3, 131-137.

Yoshimura, H., Dhar, D.K., Nakamoto, T., Kotoh, T., Takano, M., Soma, G., and Nagasue, N. (2003). Prognostic significance of tumor necrosis factor receptor in colorectal adenocarcinoma. Anticancer Res 23, 85-89.

Zhao, X., Rong, L., Li, X., Liu, X., Deng, J., Wu, H., Xu, X., Erben, U., Wu, P., Syrbe, U., et al. (2012). TNF signaling drives myeloid-derived suppressor cell accumulation. J Clin Invest 122, 4094-4104. 